\title{
In Contact Observation of Model Synovial Fluid Lubricating Mechanisms
}

Connor Myant* and Philippa Cann

Tribology Group, Department of Mechanical Engineering, Imperial College London, SW7 $2 A Z$

*Corresponding author: connor.myant@imperial.ac.uk

\section{ABSTRACT}

This paper examines the fundamental mechanisms of synovial fluid lubrication in artificial joints. Film thickness measurements were made for bovine serum solutions in a model test device. In contact imaging was also carried out to aid interpretation of these results. The results indicated that two types of film are formed; a boundary layer of adsorbed protein molecules, which are augmented by a high-viscosity fluid film generated by hydrodynamic effects. The high-viscosity film is due to inlet aggregation of protein molecules forming a gel which is entrained into the contact preferentially at low speeds. As the speed-increases this gel appears to shear thin giving much lower lubricant film thickness. Results suggest that protein-containing fluids do not obey classical Newtonian EHL models.

Key words: Artificial hip joints; boundary lubrication; synovial fluid; film thickness

\section{List of Abbreviations}

BCS Bovine calf serum

BL Boundary lubrication

EHL ElastoHydrodynamic lubrication

$\lambda \quad$ lambda ratio $=$ minimum film thickness/surface roughness $(\mathrm{Ra})$

MoM Metal-on-Metal

MoP Metal-on-Polyethylene

SF Synovial fluid

SRR Slide-roll ratio 


\section{INTRODUCTION}

Artificial hips experience complex kinematic and loading patterns during use, which coupled with the varied physical and chemical properties of lubricating synovial fluid (SF) represents a very difficult tribological problem. Failure of both MoM and MoP joints has been linked to excessive wear of components due to poor tribology. Damage of the rubbing surfaces is due to physical (abrasion or adherence) or chemical (corrosion) wear mechanisms resulting in the formation of micron (polymer) or nanometre (metal) sized debris. Wear is essentially determined by the properties of the implant surfaces and the nature (chemical and physical) of the lubricating film. In recent years there has been considerable research into material wear in both model and hip simulator studies [1-4], however there are relatively few studies of SF lubrication mechanisms and film properties in the literature.

Two distinct mechanisms: $E H L$ and $B L$ are usually described in the literature. $E H L$ theory suggests film formation in articular joints is dominated by entrainment and squeeze effects $[5,6]$. These models have been used to predict film thickness over the gait cycle using a very simple isoviscous, continuum fluid model. The results suggest that for MoM hips the contact operates in the mixed regime $(\lambda<3)$ for at least part of the gait cycle [6]. In the EHL models film thickness is essentially determined by the bulk viscosity and mean speed of the articulating surfaces. EHL theory also predicts film thickness is relatively insensitive to pressure [7]. BL theories of SF lubrication propose that the articulating surfaces are protected by a thin adherent adsorbed or reacted film. Different components of SF have been identified as boundary additives including proteins, glycoproteins and phospholipids [8, 9].

Recent research carried out by the authors has examined the fundamental mechanisms of model SF. Initial results [10] showed that for simple protein containing solutions in model sliding contacts friction and film thickness were dependent on protein content and lubricant chemistry. Fan et al [11] suggested that film formation was determined by an aggregation mechanism which controlled protein behaviour in the inlet of the contact. In a new paper [12] the authors concluded protein-containing solutions demonstrate complex timedependent film thickness behaviour that is not characteristic of a simple Newtonian fluid. An aggregation mechanism, due to flow effects in the inlet, created a new gel-like protein phase. This protein gel formed an inlet reservoir feeding the contact with high viscosity lubricant generating larger than predicted film thickness. Proteins which passed through the contact formed thick deposited films on the metallic surface. This observation is supported by other 
studies [13-15] which reported thick protein deposits on joint surfaces after in vitro and in vivo (explant) use. It is usually suggested that these films act as a protective surface layers reducing adhesion and wear [15]. The proposed lubrication mechanism is very different to classical EHL models; larger than predicted films were observed. This was tentatively linked to a change in the rheological properties of the lubricating fluid local to the contact and the formation of thick boundary layers.

Currently, computational analysis of articular joints assumes that the lubricating SF behaves as a Newtonian fluid throughout the gait cycle, allowing the lubricant to be based on an isoviscous, incompressible physical model [6]. Such models assume articular joints predominantly operate within the EHL regime. It should be noted that within this lubrication regime film thickness is determined by the viscosity of the material in the contact inlet [16]. However, the lubricant rheological properties employed in numerical predictive models are based on the bulk solution and not the fluid present in the inlet. The film thickness findings discussed above [12] showed that for protein suspensions a complex multi-mode regime exists during sliding, which leads to chaotic film distribution dependent upon the properties of the fluid local to the contact. This has large implications on numerical predictive models which at present are used as a basis for developing new hip implant designs which is dangerously simplistic. These models may lead to incorrect component design due to poor understanding of the tribological mechanisms and over simplified lubricant behaviour.

If the accuracy of artificial joint computational models are to be improved, and therefore the longevity and performance of the artificial joint; it is important to understand the mechanisms, local to the contact, which control the rheological properties of the lubricating film. The work thus far has been confined to film thickness measurements to support the concept of an inlet aggregation mechanism and the formation of a gel-phase [12]. In the current paper further film thickness data is presented but the main focus is in-contact imaging which is used to explain the proposed film formation mechanisms in more detail.

\section{EXPERIMENTAL}

\subsection{Film thickness measurement and in-contact imaging}

Lubricant film thickness was measured using a commercial optical interferometric tribometer, (Ultra-Thin Film Measurement System PCS Instruments, UK). The standard steel ball specimen was replaced with an "as cast" commercial grade CoCrMo resurfacing femoral head (38 mm diameter). This was held in the test device and loaded against the underside of the rotating glass disc. The head was held in a supporting mount (Fig. 1) and could be 
rotated so that a different position was used for each test. The femoral head was held stationary, allowing tests to operate in pure sliding conditions. A copious supply of lubricating fluid was pumped onto the femoral head and entrained into the contact by rotation of the glass disc. A thick liquid layer on the glass disc was maintained throughout the test period to avoid effecting contact film thickness through evaporation. The underside of the glass disc was coated with a thin chromium layer $(\sim 10 \mathrm{~nm})$ overlaid by silica $(\sim 500 \mathrm{~nm})$; this provides the necessary reflection condition to measure the film thickness in the glass/metal interface. Single-position film thickness measurements from the centre of the contact were obtained using the spectrometer. Further details of this method may be found in [12]. For some tests the spectrometer system was replaced by a CCD camera and the contact zone observed directly so development of the interfacial film could be followed.

The test specimens were cleaned ultrasonically in $1 \%$ sodium dodecyl sulphonate solution in deionised water. The specimens were then rinsed three times in deionised water and finally washed in Analar isopropanol. Before the test specimens were air-dried and then mounted in the optical device, which was held at $37^{\circ} \mathrm{C}$ for 1 hour prior to testing. The test solution was fed directly into the contact zone using a syringe to prevent contamination. Lubricant supply was maintained throughout the test period to insure a substantial fluid meniscus around the contact zone. The test conditions (sliding seed, contact pressure) were chosen to be representative of those occurring during the gait cycle in MoM hip joints [12].

\subsection{Test programme}

The work has concentrated on film formation by protein-containing solutions. The test programme was as follows:

1. Film formation under cyclic loading at constant speed $(0,10 \mathrm{~mm} / \mathrm{s})$ : The contact was loaded $(5 \mathrm{~N}$ ) for approximately $15 \mathrm{~s}$ while film thickness measurements were taken and then un-loaded for approximately $45 \mathrm{~s}$, this was repeated every minute for test period (12 minutes). Fresh fluid was continuously supplied to the contact. Film formation at zero and a mean speed of $10 \mathrm{~mm} / \mathrm{s}$ was measured.

2. Film formation as a function of sliding speed: At the end of the constant speed test a speed-sweep test was carried out over the range $5-50 \mathrm{~mm} / \mathrm{s}$. Film thickness readings were taken periodically over the entire speed range.

3. In some experiments colour interferograms were captured using a 3CCD RGB camera (CV M9CL, JAI). A magnification of $5 \mathrm{X}$ (objective) was used to observe both the Hertzian contact region and inlet/outlet areas. 


\subsection{Test lubricants}

BCS (Sigma Aldrich 12133C protein concentration $72 \mathrm{mg} / \mathrm{ml}$ ) was used as the model SF in all tests it was diluted to $25 \%$ w/w with distilled water (Sigma-Aldrich, S-37531-356). BCS contains primarily a mixture of albumin and $\gamma$-globulin proteins. No additional buffers or antibacterial agents with used. All fluids were stored at $5{ }^{\circ} \mathrm{C}$ and used within a few days of preparation.

\section{RESULTS}

Initially film thickness was studied as a function of time under cyclic loading $(U=0)$ and constant load pure sliding $(U=10 \mathrm{~mm} / \mathrm{s})$ conditions. Figure 2 presents typical spectrometric film thickness measurements for BCS plotted against time/load cycle number. For the cyclic load investigation one minute represents a loading and un-loading cycle. Under cyclic loading conditions a thin film (5-10 nm) was quickly formed and maintained throughout the test period. This result was found to be very repeatable. Sliding tests induced a thick film, this lubricant film was observed to be less stable and some scatter was found in repeats. However, the trend of an increasing film over the entire test period was always observed. A final film thickness value of ca $30 \mathrm{~nm}$ was recorded.

Figures 3 and 4 show selected interference images of the contact zone at $t=0,6$ and 12 minutes during the test. The contact zone is seen in the centre of the picture surrounded by the interference fringes which indicate increasing surface separation. At the start of the test the contact is a green/blue colour which is due to the silica spacer layer. Under cyclic loading conditions a thin protein surface layer was observed to form after a few loading cycles, represented by the dark brown areas. These areas are distributed un-evenly over the contact area but were observed to initiate at asperity sites. No wear to the CoCrMo surface was observed during cyclic loading, however, considerable wear did occur during sliding (constant load). This can be seen in Fig 4 as a large increase in the contact area. A new fluid phase can also be seen in the inlet zone represented by dislocations in the fringes, caused by a change in the fluid's refractive index. Periodically the gel-phase is entrained into the contact area locally increasing the film thickness. The new inlet phase was observed to form rapidly within a few seconds and increase in length with time. The inlet phase, and subsequent thick protein layer within the main contact area, was easily removed or disturbed by scratches on the glass disc.

The second series of experiments recorded film thickness as a function of speed. Earlier speed-sweep measurements showed complex time-dependent effects which made the 
results difficult to interpret [11]. This is thought to be due primarily to the changing contact pressures because of the rapid wear of the femoral head. In the current study speed-sweep measurements were obtained immediately after the constant speed tests. At this point in the test the wear scar had formed and because of the reduced contact pressure, the film thickness has stabilized. Figure 5 presents central film thickness results plotted against sliding speed for the range $0-60 \mathrm{~mm} / \mathrm{s}$. Film thickness is observed to fall with increasing sliding speed. The thickest films are formed at the lowest speeds, which represents nonclassical behavior and is opposite to EHL predictions [7].

Figure $6 \mathrm{~A}$ to $\mathrm{H}$ shows selected interference images of the contact zone during sliding at $U=$ $0,3,8,10,20,30,40,50 \mathrm{~mm} / \mathrm{s}$ respectively. The new inlet protein phase is more noticeable compared to Fig. 4; fringe dislocations are more apparent. A close up view of the contact inlet from Fig. $6 \mathrm{C}$ is shown in Fig. 7. The length of the inlet reservoir was observed to vary with sliding speed, the smallest lengths seen at the lowest sliding speeds $(U=0-5 \mathrm{~mm} / \mathrm{s})$. A maximum reservoir length, several times the contact radius, was observed at a sliding speeds of ca $15 \mathrm{~mm} / \mathrm{s}$. Before decreasing in length to less than the contact radius, around $U=40-50 \mathrm{~mm} / \mathrm{s}$. Material, possibly proteins, can also be seen exiting the contact as small black spots at the rear of the contact. The amount of expelled material increases with sliding speed.

At the end of the test the femoral head was dismounted, rinsed lightly with water and the wear scar examined under a low power microscope (an example is shown in Fig. 8). Figure $8 \mathrm{~A}$ shows the femoral head in an un-worn state prior to testing, while Fig. 8B shows the femoral head after testing and post rinsing. The wear scar is seen as a polished region in the centre of the photograph. Deposited gelatinous material is observed in the inlet.

\section{DISCUSSION}

Spectrometric film thickness measurements and in contact imaging display a complex lubrication mechanism for the model SF lubricated contact. Time and shear rate dependent behavior was observed. Two different mechanisms of film formation were recorded:

1. Deposition of a thin, boundary film under cyclic loading.

2. Formation of a gel-phase in the inlet region which is entrained into the contact and contributes to thicker than predicted films at low speeds. Entry of this protein phase into the contact is complex and shows time and shear rate dependency. 


\subsection{Boundary film formation}

Protein absorbance at the metal-liquid interface has been extensively reported particularly for albumin [17] containing solutions. Under cyclic loading conditions a small thin surface layer can be seen within the contact, $\sim 5 \mathrm{~nm}$ in thickness. The protein layer formed relatively quickly and after only a few loading cycles. This suggests that the proteins readily deposit onto the CoCrMo surface, indeed this has been observed previously for metallic surfaces [18]. Large protein deposits were only visible on the hydrophobic CoCrMo surface and not the hydrophilic glass disc. It has been reported that most proteins preferentially absorb onto hydrophobic rather than hydrophilic surfaces [19] which explains this observation. The cyclic loading may aid, or accelerate, this deposition process to form robust protein films by increasing protein-protein interaction, transportation of proteins to the surface and rearranging or reorientating the absorbed protein structure [12]. The protein film was not distributed evenly and was observed to form, initially, around the "blocky carbide" sites. This could be a result of preferential absorbance to the different local metallurgy of the femoral head particularly the area around the "carbides", which is reported to have a lower chromium content [19]. This local deposition of proteins has significant implications for the surface treatment of the CoCrMo used for MoM joints. The femoral head used in this investigation was as-cast, which leaves large carbide precipitates on the surface. Heat treated components remove these asperities [19]. Further investigation is required to determine what effect this may have.

A thick lubricant film is also observed in the constant speed tests, where film thickness increased with time (sliding distance) throughout the test period. However, rapid wear of the CoCrMo surface occurred, significantly increasing contact size and thereby reducing the contact pressure (Figs 4 and 6). One explanation for the gradually increasing film is the decreasing contact pressure rather than deposition of surface films [12]. There was little evidence that the femoral head wear was abrasive or a direct result of material entrainment. In some cases scratches were observed and this is thought due to entrainment of "carbide" particles which were rapidly removed from the rubbing surfaces. If an abrasive mechanism was the main wear mechanism this should have been visible on both of the contacting surfaces. However, no wear to the, softer, glass disc was observed. At this stage it is not clear if this phenomenon was caused by the proteins or perhaps driven by a corrosive mechanism.

The rapid increase in film thickness with decreasing contact pressure has considerable implications for MoM lubrication. The new generation MoM joints which have increased 
head sizes and reduced clearance operate under much lower contact pressures compared to the small-head designs [20]. This will result in much thicker lubricant films and hence improved wear resistance. The observed film thickness pressure-sensitivity would also suggest the films are compressible and elastic. However these films will be exceptionally susceptible to local increases in pressure; for example with edge-loaded components and this may be the cause of local severe wear associated with high inclination cup positions [21].

\subsection{Protein gel formation and contact entry}

When sliding occurred the observed film thickness behavior appeared to be governed by the formation of a new inlet phase. The size of the new inlet phase was found to exhibit speed dependency. Figure 9 shows central film thickness and inlet reservoir length plotted against entrainment speed. Inlet reservoir length, $s$, has been normalized by the contact radius, $a$, so that when $s=1=a$. A clear relationship between inlet length and central film thickness is observed; suggesting that film thickness is determined by the viscosity and amount of material in the contact inlet. However it should be remembered that the contact movement is confined to simple uni-directional sliding with constant load. In reality hips undergo bidirectional sliding with multiple flow axes and transient loading conditions. The reversal in flow direction and change in axis may slow, or prevent, the buildup of the inlet aggregates; this is currently under investigation.

Inlet aggregation behavior has been reported previously for suspended material in both hard [22-25] and soft lubricated contacts [26]. In this work the ability of the suspended proteins to form inlet aggregates and then pass through the contact is controlled by a combination of contact conditions (load, speed, SRR) and lubricant properties (protein concentration, size and morphology). The majority of articular joints operate in pure sliding conditions, which promotes inlet aggregation [22], due to the force couple which would tend to cause the particles to rotate. In a rolling contact the forces would drag the particles through the contact. In pure sliding inlet aggregation allows a new protein enriched gel-like phase to form; the size of this new inlet reservoir is time and speed dependent. At low speeds fluid forces are small and surface drag forces dominate contact entry, causing more proteins to enter the contact [23]. The observed increase in film thickness with decreasing entrainment speed may be a result of this force balance. Figure $6 \mathrm{~B}$ shows chaotic (un-even) film thickness distributions at low speeds as small protein agglomerates are dragged through the contact. Friction forces between the protein aggregates and main contacting bodies are large compared to the hydrodynamic forces, causing rapid entrainment of any protein 
aggregates present in the inlet region. As entrainment speed is increased the contact inlet is fed with proteins at an increasing rate, causing the new inlet reservoir to grow and become visible. However hydrodynamic forces also increase causing a greater degree of contact evasion for smaller proteins [24]. The larger protein-aggregates which are left in the inlet reservoir require greater frictional forces to drag them into the contact [25], causing the film thickness to decrease. As the sliding speed is increased further the hydrodynamic forces are large enough to sweep large protein-aggregates around the outside of the contact, causing a reduction in the inlet reservoir size and a further reduction in the lubricating film thickness (Fig. 5).

The contact, due to the geometry and SRR, acts to collect suspended molecules in the inlet zone. At this stage it is un-known which components within the BCS solutions are drawn into the contact and which are filtered out over the shear rates tested. The individual proteins have been reported to be ca $1-5 \mathrm{~nm}$ in size, whilst it is known that proteins aggregate both in solution, under shear, at surfaces and after heat treatment [17]. Once these protein aggregates collect in the contact inlet zone the overall size may be much larger than the central contact film thickness. For hard non-complaint particles in metallic bearing contacts there is a maximum size of particle that can be entrained into a contact due to geometry [24]. This is unlikely to be a problem with protein aggregate entrainment, as these will easily deform, allowing for very large aggregates to be entrained. As the protein aggregates enter the contact they undergo very high shear rates $\left(>10^{5} \mathrm{~s}^{-1}\right)$, they are squashed and stretched. These proteins are forced out of solution and possibly de-natured. A thick deposited layer is formed within the contact which is thought to protect the femoral head from wear $[12,15]$. However we have observed this layer to be fragile and easily removed when disturbed; a sudden change in load or the passing through of a scratch completely removed the protein film. During post-test analysis these layers dried and were observed to become robust and highly adherent to the femoral surface. Even after rinsing of the CoCrMo surface a protein deposit was still visible in the inlet region (Fig 8B).

BCS has been used extensively in the literature as a model SF fluid and is known to exhibit shear and time dependent rheological properties. At very low, constant, shear rates $\left(<1 \mathrm{~s}^{-1}\right)$ BCS is thought to be rheopectic [28]. As shear rates increase the fluid shear thins [29], until, at very high shear rates $\left(>10^{3} \mathrm{~s}^{-1}\right)$ a $2^{\text {nd }}$-Newtonian phase is reached [30]. This shear rate dependent rheology can be explained by the inlet aggregation and contact entry mechanism presented in this paper. At very low, or constant, sliding speeds (low inlet shear) protein aggregates have sufficient time to form in the inlet, feeding the contact with a highly viscous, 
protein enriched fluid. Figure 2 shows that at constant speed film thickness continues to increase with time. One suggestion for this effect is the reduction in contact pressure due to wear of the femoral head. An alternative explanation is that film-growth is due to an increasing rheopectic-like response. As the entrainment speed (shear rate) increases so does contact evasion of the protein aggregates in the inlet zone, due to an increase in the fluid forces. Larger proteins are left in the inlet reservoir, which require greater drag forces to enter the contact. Film thickness begins to drop, giving the appearance of a shear thinning fluid. At this stage it is unknown whether the viscosity of the protein-gel phase decreases, or alternatively, it is now harder for the protein aggregates to enter the contact. In other words; thinning of the suspension medium or reduction in protein contact entry would cause a lower effective viscosity. At very high entrainment speeds (high shear rates) only the largest proteins are left in the inlet zone. The new inlet reservoir size plateaus and the contact is fed with a constant supply of large proteins, giving the appearance of a $2^{\text {nd }}$-Newtonian phase.

In classical EHL theory the contact film thickness is determined by the properties of the material in the contact inlet [16]. For the range of typical geometries and speeds experienced by an artificial hip over a gait cycle the shear rate, within the inlet region, will be in the range of $10^{2}-10^{4} \mathrm{~s}^{-1}$. This suggests the BCS within the inlet operates in the shear thinning regime and this agrees with the film thickness results presented in Fig. 5. Whilst under constant shear the BCS time dependent nature is rheopectic. Describing BCS as rheopectic in hip joints seems a little confusing at first; as the liquid is flowing through a convergent wedge and therefore must be undergoing increased shear. It is the aggregation of the proteins, due to the contact geometry, and the formation of large protein networks [28] which produce the rheopectic-like behavior. The conclusion from this is that any rheological changes are local to the contact region and do not occur throughout the bulk lubricant. Great care should be taken when describing the rheological behavior of complex suspension fluids such as BCS and SF in bearing-like contacts.

It would be advantageous to be able to employ the film thickness data to determine quantitatively how the rheology of the protein solution and, thus, the fluid in the contact inlet varies with distance from the solid surfaces. Recently there have been some attempts in the literature [31], however these rely on conventional EHL film thickness equations which are only applicable to systems where there is a significant degree of increase in the inlet viscosity [32]. BCS is essentially an aqueous suspension and the viscosity of water is low, but more importantly the pressure viscosity coefficient is tiny [32]. Other equations have been developed to predict film thickness for such fluids, or when contact pressures are not 
large enough to cause a viscosity change; such as highly conformal contacts or elastomeric bearings $[6,33,34]$. Hooke provides the following central film thickness, $h$, equation for elastic-isoviscous contacts [34];

$$
h=4.18 \frac{(U \eta)^{0.6} R^{10.67}}{W^{0.13} E^{\prime 0.47}}
$$

where $U$ is the entrainment speed, $\eta$ is the viscosity of the lubricant; $R^{\prime}$ is the reduced radius, which for this case is the radius of the femoral head; $W$ is the applied load and $E^{\prime}$ is the reduced Young's modulus defined by $2 / E^{\prime}=\left(1-v_{1}{ }^{2}\right) / E_{1}+\left(1-v_{2}^{2}\right) / E_{2}$, respectively, where $R_{x 1}, R_{x 2}, E_{1}, E_{2}, v_{1}$, and $v_{2}$ denote the radii in the entrainment direction, the Young's moduli, and the Poisson's ratios of the two contacting bodies.

Figure 10 presents experimental and theoretical central film thickness results for BCS versus entrainment speed. The dotted line represents the values produced by Eq. 1 if the viscosity is assumed constant. A value of $\eta=0.0025$ Pas was employed, this was taken from refs [6, 30] and is suggested as an appropriate viscosity during articulation [6]. It is clear from Fig. 10 that such a simple estimation of the lubricant viscosity predicts thinner films compared to those measured experimentally. A good fit can be achieved if the viscosity component, $\eta$, is altered by $d h$ so that the theoretical $h$ from Eq. 1 matches the experimental results (plotted as a solid black line). The viscosity was found to vary, with respect to entrainment speed, by $\eta=k U^{z}$, where $k$ and $z$ are the viscosity power law coefficient and index, respectively. The use of a power law model is justified by the observation that the viscosity is linear on a loglog scale, shown in Fig. 11. A good fit was found for $k=0.0007$ and $z=-1.25$. The results suggest that the lubricant viscosity is much greater than previously reported [6, 28-30]. At this point it should be noted that this is a crude system for calculating viscosity as it assumes a single phase fluid. It should only be used as a tentative indication of the true viscosity. The high viscosity values are an indication that the film thickness is due to the mechanical properties of the protein networks and not the suspension medium, or bulk phase, rheological properties. This system assumes the only error in Eq. 1 is due to the viscosity component. The mechanical properties of the protein networks (protein gel) and protein boundary layers will vary in depth, which should be represented in the load [12] and reduced Young's modulus components. To calculate a more accurate viscosity probably requires a full 2-D solution of the point contact EHD problem, one which varies the lubricant properties with distance from the contacting surfaces. An empirical model is the subject of a current investigation, using AFM and fluorescence techniques to obtain rheological characteristics of 
the protein aggregates. This will be reported in further detail in a later paper.

The full implications of the results from this study for the design of artificial hip joints are not yet clear. Further investigation is required to determine the existence of an inlet aggregation system in transient contact conditions. However, the formation of a protein boundary layer and the protective role that it plays [12] has large implications for the selection of component material pairing; i.e. the change in surface hydrophobicity with ceramic-on-ceramic, or more likely metal-on-polymer artificial joints. The authors hope to extend the current research to cover these material pairs, however, some experimental development is required. Numerical models are commonly used to aid development and design of artificial hip joints. The results presented in the paper suggest that the current models, which are based on a simple rheological model, are inaccurate and may lead to poor component design. It is clear that before accurate numerical film thickness and wear predictions can be achieved a full rheological model of the lubricant, local to the contact during articulation, must be determined.

\section{CONCLUSION}

This paper reports on the film formation mechanisms observed in a model MoM hip joint lubricated with bovine serum. It has been shown that model SF solutions demonstrate complex time-dependent film thickness behavior that is not characteristic of a simple Newtonian fluid. The contact acts to collect proteins within the inlet region, forming a new protein enriched phase. The subsequent contact film thickness is controlled by the rheological properties of this new inlet reservoir and not the bulk solution. The new proteingel phase has a much higher viscosity than the bulk solution. Consequently, a significantly thicker film is formed than would be predicted by classical EHD theory. Contact entry of this protein phase is complex and dependent on a number of contact conditions (speed, load, geometry) and lubricant properties (size, concentration).

\section{ACKNOWLEDGEMENTS}

The authors wish to thank the EPSRC for funding this research (EP/E028721/1).

\section{REFERENCES}

[1] Wroblewski, B. M. and Siney, P. D. Charnley low- friction arthroplasty of the hip-long term results. Clin. Orthop. Related Res., 1993, 292; 191-201

[2] Revell, P.A. Al-Saffar N. and Kobayashi, Biological reaction to debris in relation to joint 
prostheses. Proc Instn Mech Engrs Part H 1997, 211, 187-197

[3] Katti, K.S., Biomaterials in total joint replacement. Colloids and Surfaces B: Biointerfaces, 2004, 39; 133-142.

[4] Bell J., Tipper J.L., Ingham E., Stone M.H., Fisher J., The influence of phospholipid concentration in protein-containing lubricants on the wear of ultra-high molecular weight polyethylene in artificial hip joints. Proc Instn Mech Engrs, Part H. 2005, 215; 259-263.

[5] Jin, Z. Dowson, D. Elastohydrodynamic Lubrication in Biological Systems. Proc Instn Mech Engrs Part J. 2005, 219; 367-380.

[6] Dowson D, Jin Z. Metal-on-metal hip joint tribology. Proc Instn Mech Engrs Part H. 2006, $220 ; 107-118$.

[7] Esfahanian M, Hamrock B J. Fluid film lubrication regimes revised. Tribology Transactions. 1991, 34(4); 628-632.

[8] Gale, L.R., Chen, Y., Hills, B.A. and Crawford, R. Boundary lubrication of joints Characterization of surface-active phospholipids found on retrieved implants. Acta Orthopaedica. 2007, 78; 309-314.

[9] Swann D A, Radin E L, Nazimiec M, Weisser P A, Curran N, Lewinnek G. Role of hyaluronic acid in joint lubrication. Ann Rheum Dis. 1974, 33; 318-26.

[10] Mavraki, A. Cann P. Friction and lubricant film thickness measurements on simulated synovial fluids. Proc Instn Mech Engrs Part J. 2009, 223(3); 325-335.

[11] Fan, J. Myant, C. Underwood, R. Cann, P. M. Hart, A. Inlet protein aggregation: a new mechanism for lubricating film formation with model synovial fluids. Proc Inst Mech Engrs Part H. 2011, 225; 696-709.

[12] Myant, C. Underwood, R. J. Fan, J. Cann, P. M. Lubrication of metal-on-metal hip joints: The effect of protein content and load on film formation and wear. Journal of the Mechanical Behavior of Biomedical Materials. 2011. doi:10.1016/j.jmbbm.2011.09.008

[13] Wang, A., Essner, A., and Schmidig, G. The Effects of Lubricant Composition on in Vitro Wear Testing of Polymeric Acetabular Components. J. Biomed. Mater. Res. Part B. Appl. Biomater. 2003, 68(B); 45-52.

[14] Scholes, S.C, Unsworth, A. and Goldsmith, A.A.J. A frictional study of total hip joint 
replacements. Phys. Med. Biol. 2000, 45; 3721-3735.

[15] Wimmer M.A. Sprecher C., Hauert R. Täger G. and Fischer A. Tribochemical reaction on metal-on-metal hip joint bearings. A comparison between in-vitro and in-vivo results. Wear 2003, 255; 1007-1014.

[16] Wedeven, L. D. Evans, D. Cameron, A. Optical analysis of ball bearing starvation. ASME Jour Lubr Tech. 1968, 90; 580-591

[17] Santos, O, Nylander, T. Paulsson, M. and Trägårdh, C. Whey protein adsorption onto steel surfaces - effect of temperature, flow rate, residence time and aggregation. J. Food Eng. 2006, 74; 468-483.

[18] Malmsten, M. Formation of absorbed protein layers. Journal of Colloid and Interface Science. 1998, 207; 186-199.

[19] McMinn, D. Modern hip resurfacing. Springer-Verlag London Limited. 2009

[20] Liu, F. Jin, Z, Roberts, P. Grigoris, P. Importance of head diameter, clearamce, and cup wall thickness in elastohydrodynamic lubrication analysis of metal-on-metal hi resurfacing prostheses. Proc Inst Mech Engrs Part H. 2006, 220; 695-704.

[21] Hart, A. J. Ilo, K. Underwood, R. Cann, P. Henckel, J. Lewis, A. Cobb, J. Skinner, J. The relationship between the angle of version and rate of wear of retrieved metal-on-metal resurfacings; A prospective, CT-based study. J bone joint surg br. 2011, 93(B); 315-320

[22] Wan, G. T. Y. Spikes, H. A. The behaviour of suspended solid particles in rolling and sliding elastohydrodynamic contacts. Tribology Transactions. 1988, 31(1); 12-21.

[23] Dwyer-Joyce, R. S. The effects of lubricant contamination in rolling element performance. London: University of London Press. 1993.

[24] Dwyer-Joyce, R. S. Predicting the abrasive wear of ball bearings by lubricant debris. Wear. 1999, 233-235, 692-701.

[25] Nikas, G. K. Particle entrainment in elastohydrodynamic point contacts and related risks in oil starvation and surface indentation. Journal of Tribology. 2002, 124; 461-467.

[26] Timm $\mathrm{K}$ et al. Cosmetic powder suspensions in compliant, fingerprintlike contacts Biointerphases. 2011. 6(3); 126-135. DOI: 10.1116/1.3640042 
[27] Kitano, T. Ateshian, G.A. Mow, V.C. Kadoya, Y Yamano, Y Constituents and $p H$ changes in protein rich hyaluronan solution affect the biotribological properties of artificial articular joints. J. of Biomechanics. 2001, 34; 1031-1037.

[28] Oates K, Krause WE, Jones RL, Colby RH. Rheopexy of synovial fluid and protein aggregation. J. Roy. Soc. Interface 2006, 3; 167-174.

[29] Cooke, A. V. Dowson, D. Wright, V. The rheology of synovial fluid and some potential synthetic lubricants for degenerate synovial joints. Engng in Med. 1978, 7; 66-72

[30] Mavraki, A. Cann P. Lubricating film thickness measurements with bovine serum. Tribology International. 2011, 44; 550-556.

[31] Smeeth, M. Spikes, H. A. Gunsel, S. The formation of viscopus surface films by polymer solutions: Boundary or Elastohydrodynamic lubrication?. Tribology Transactions. 1996, 39(3); 720-725.

[32] Ratoi, M. Spikes, H. A. Lubricating properties of aqueous surfactant solutions. Tribology Transactions. 1999, 42(3); 479-486.

[33] Hamrock, B. J. Dowson, D. Elastrohydrodynamic lubrication of elliptical contacts for materials of low elastic modulus 1 - Fully flooded conjunction. NASA TN D-8528 (1977).

[34] Hooke, C. J. The elstohydrodynamic lubrication of heavily-loaded point contacts. Jour Mech Eng Sci. 1980, 22, 183-187. 


\section{List of Figures}

Figure 1. Schematic of the experimental setup

Figure 2. Representative film thickness measurements for cyclically-loaded $(U=0 \mathrm{~mm} / \mathrm{s})$ and constant speed $(U=10 \mathrm{~mm} / \mathrm{s})$ tests.

Figure 3. Selected interferograms of the contact zone at $t=0(A), 6(B)$ and $12(C)$ minutes for cyclic loading tests.

Figure 4. Selected interferograms of the contact zone at $t=0$ (A), 6 (B) and 12 (C) minutes under a constant load $(5 \mathrm{~N})$ and sliding speed $(10 \mathrm{~mm} / \mathrm{s})$. The inlet is on the left of the contact region.

Figure 5. Speed-sweep results immediately after the 12 minutes $10 \mathrm{~mm} / \mathrm{s}$ test.

Figure 6. Selected interferograms of the contact zone during sliding at $U=0,3,8,10,20$, 30,40 and $50 \mathrm{~mm} / \mathrm{s}$. The inlet is at the top of the contact zone.

Figure 7. Detail of the inlet phase

Figure 8. CoCrMo Femoral head surface; A) Un-worn, surface asperities are regular and structured. B) Worn post-test, the wear scar is visible as a polished circular region and gelatinous deposit in the inlet region can also be seen.

Figure 9. Measured film thickness and inlet reservoir (gel-phase) length plotted against entrainment speed.

Figure 10. Comparison of measured and predicted film thickness (Newtonian and shearthinning models) plotted against entrainment speed.

Figure 11. Effective viscosity (estimated from film thickness results) plotted against entrainment speed. 


\section{FIGURES}

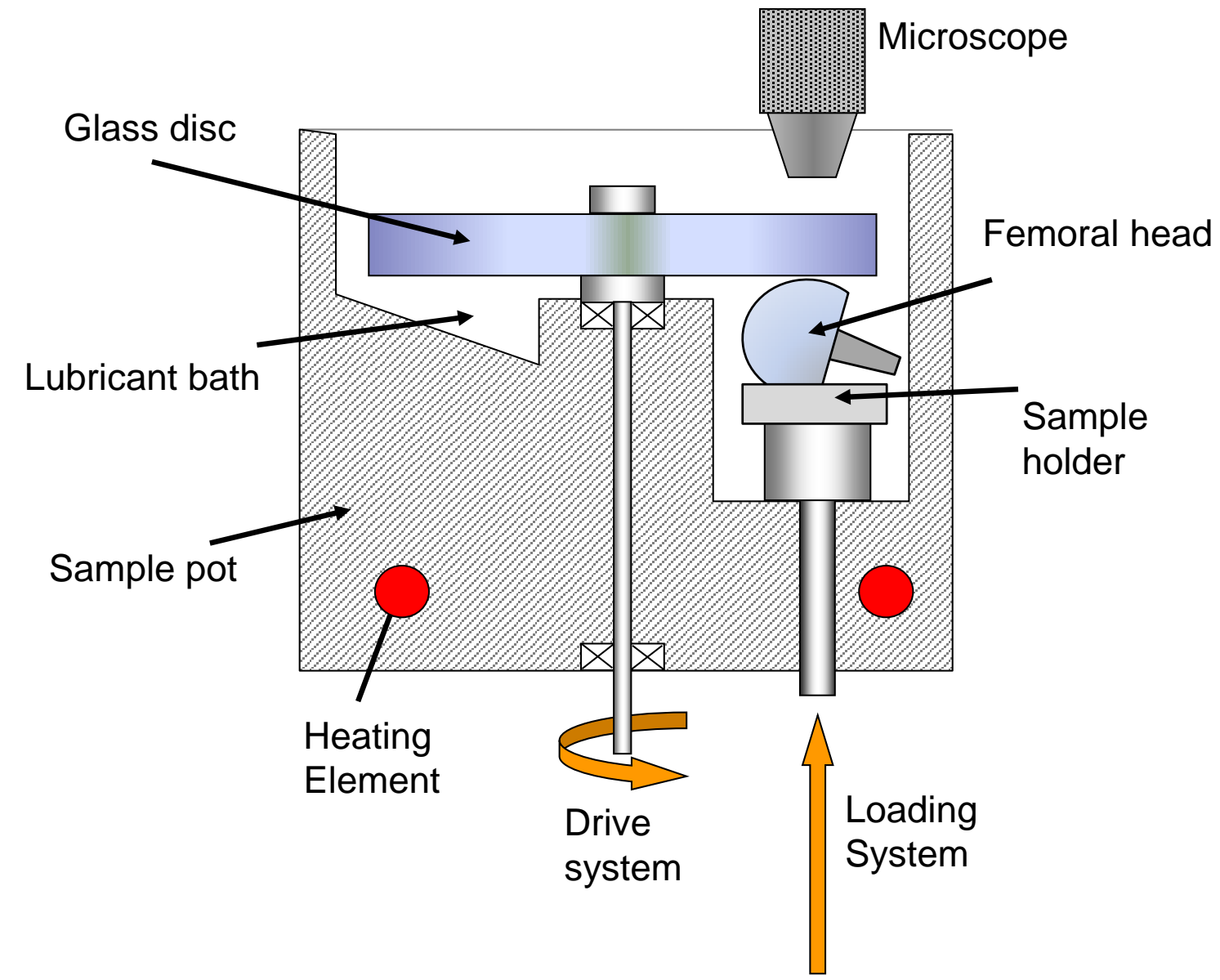

Figure 1. Schematic of the experimental setup 


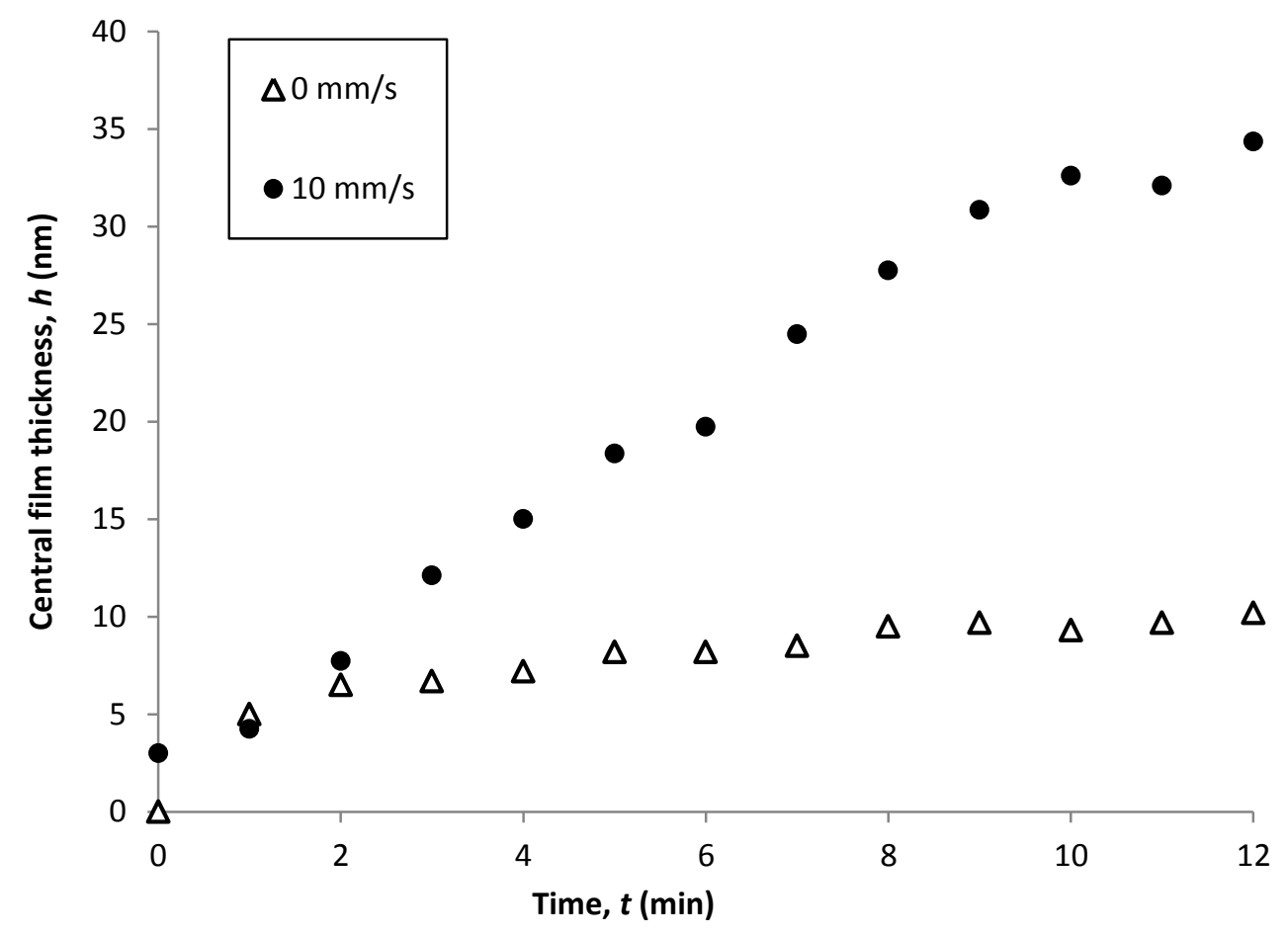

Figure 2 Representative film thickness measurements for cyclically-loaded $(U=0 \mathrm{~mm} / \mathrm{s})$ and constant speed $(U=10 \mathrm{~mm} / \mathrm{s})$ tests.
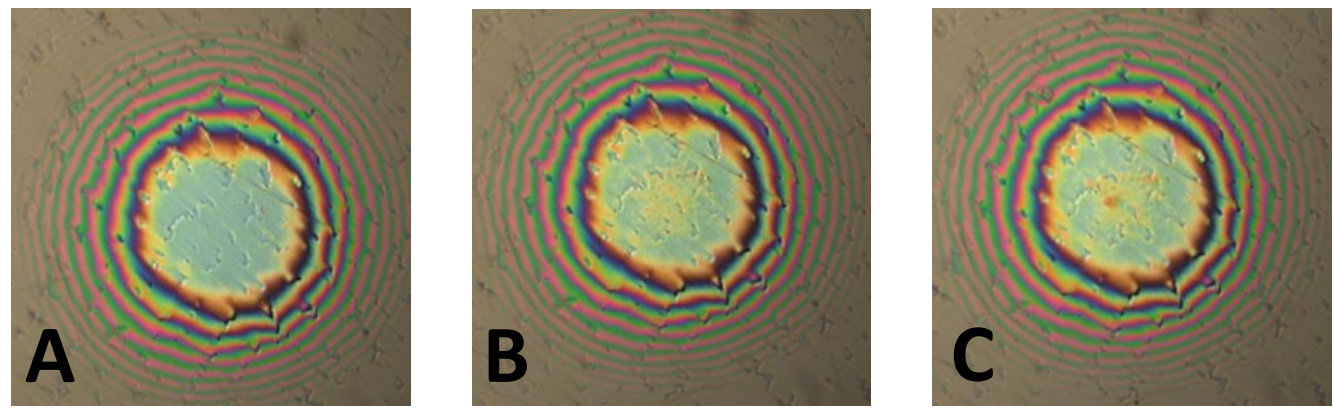

Figure 3. Selected interferograms of the contact zone at $t=0$ (A), 6 (B) and 12 (C) minutes for cyclic loading tests. 

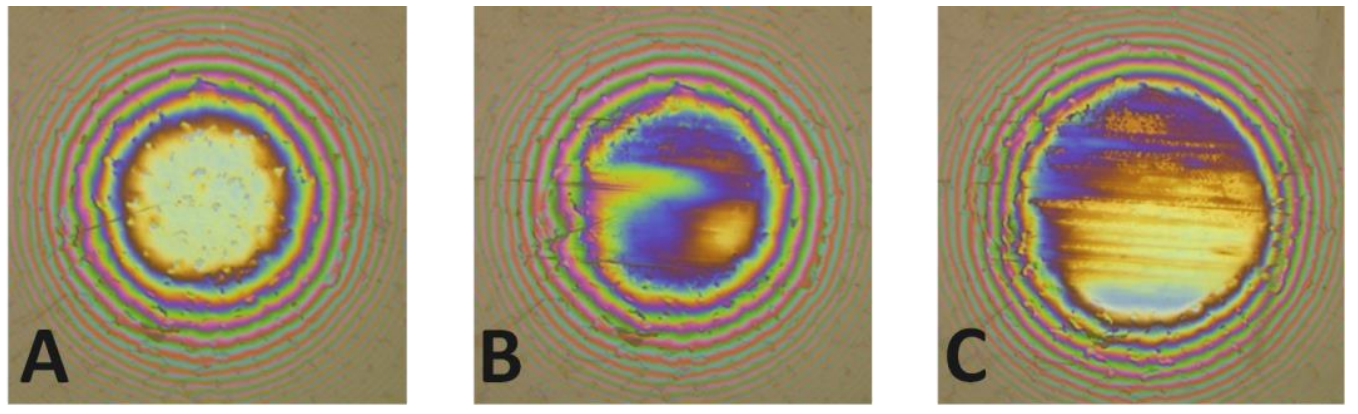

Figure 4. Selected interferograms of the contact zone at $t=0(\mathrm{~A}), 6(\mathrm{~B})$ and 12 (C) minutes under a constant load $(5 \mathrm{~N})$ and sliding speed $(10 \mathrm{~mm} / \mathrm{s})$. The inlet is on the left of the contact region.

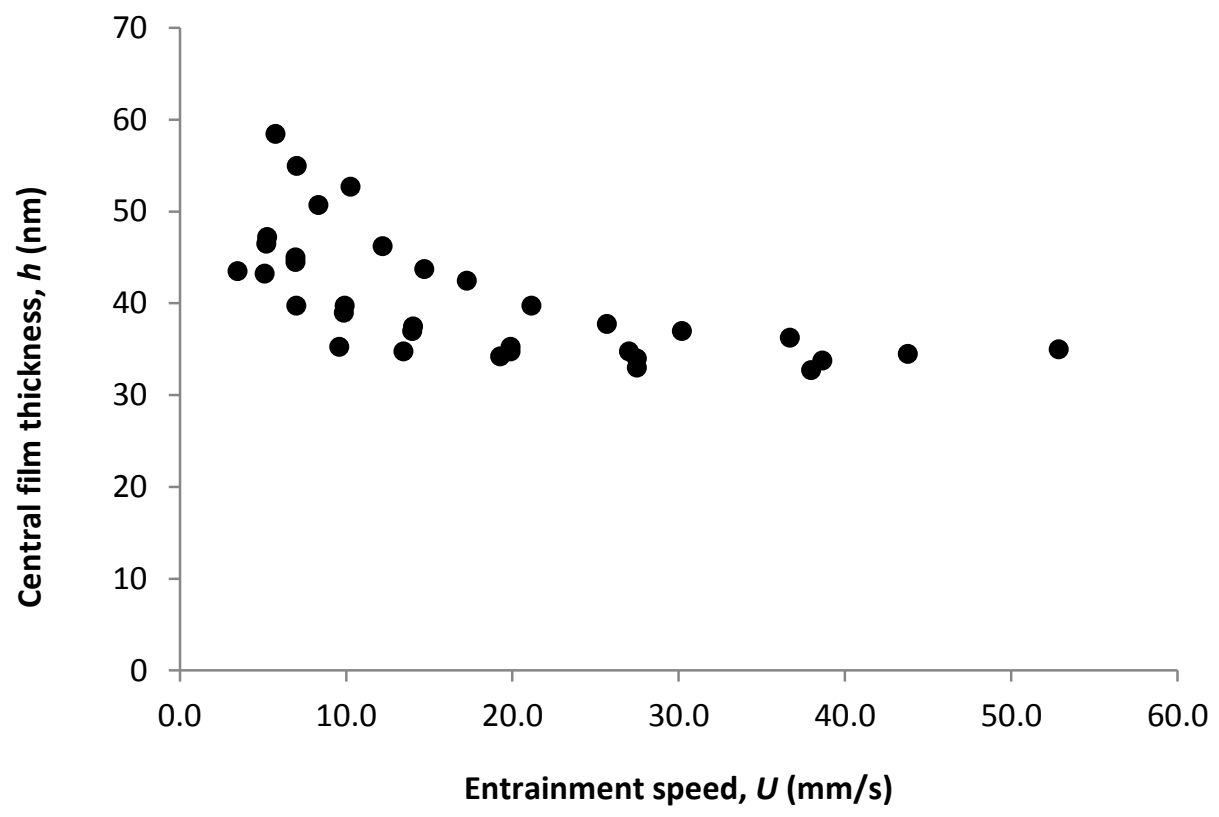

Figure 5 Speed-sweep results immediately after the 12 minutes $10 \mathrm{~mm} / \mathrm{s}$ test. 

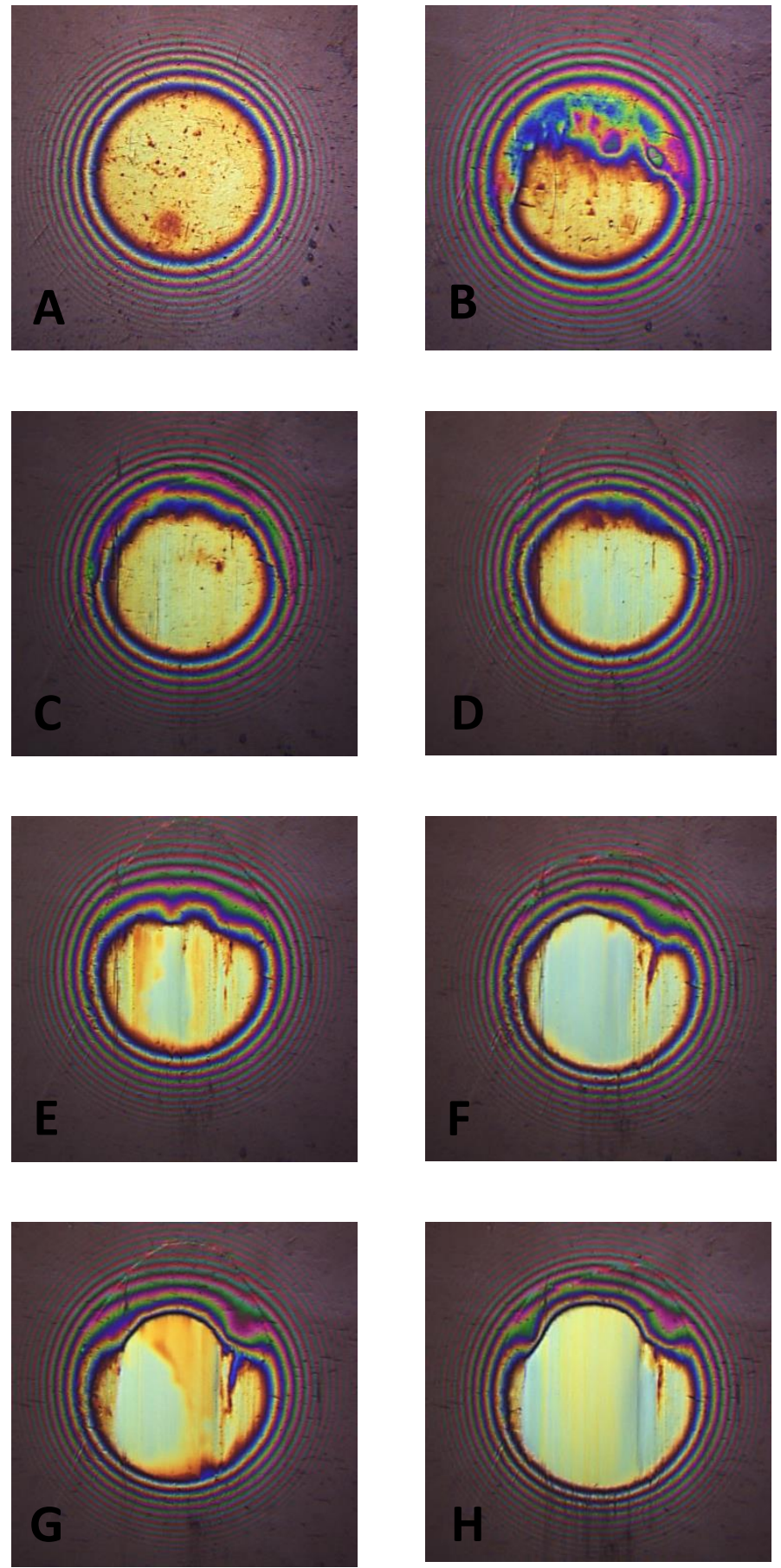

Figure 6. Selected interferograms of the contact zone during sliding at $U=0,3,8,10,20$, 30,40 and $50 \mathrm{~mm} / \mathrm{s}$. The inlet is at the top of the contact zone. 


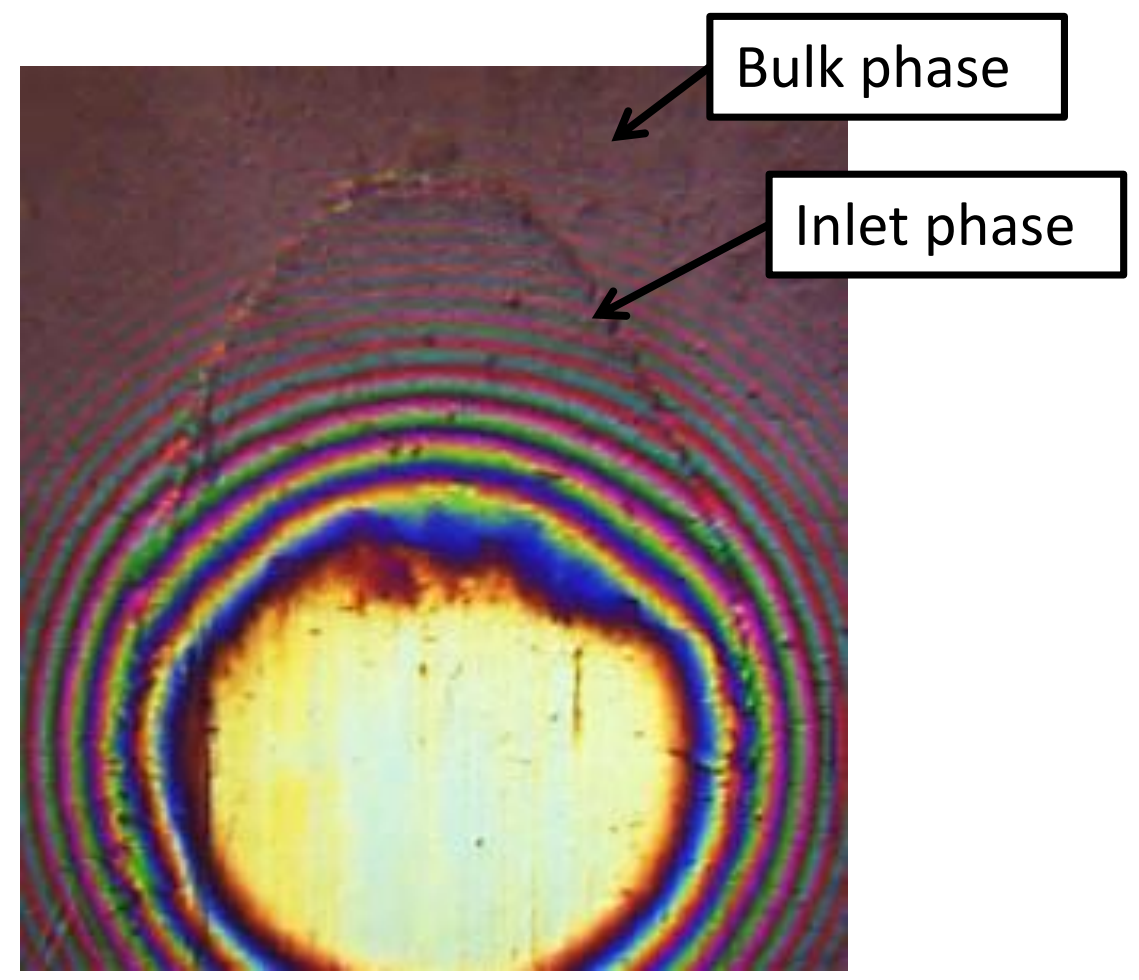

Figure 7 Detail of inlet phase
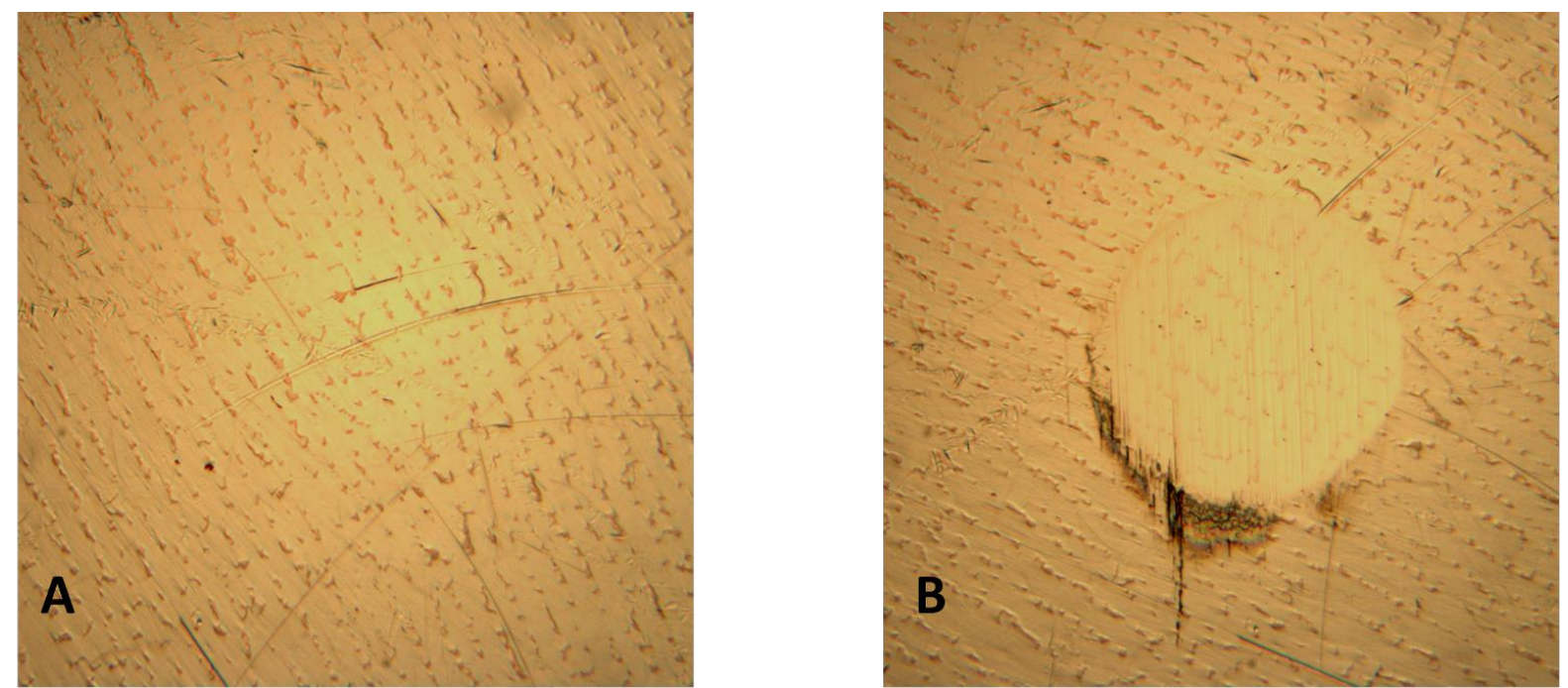

Figure 8. CoCrMo Femoral head surface; A) Un-worn, surface asperities are regular and structured. B) Worn post-test, the wear scar is visible as a polished circular region and gelatinous deposit in the inlet region can also be seen. 


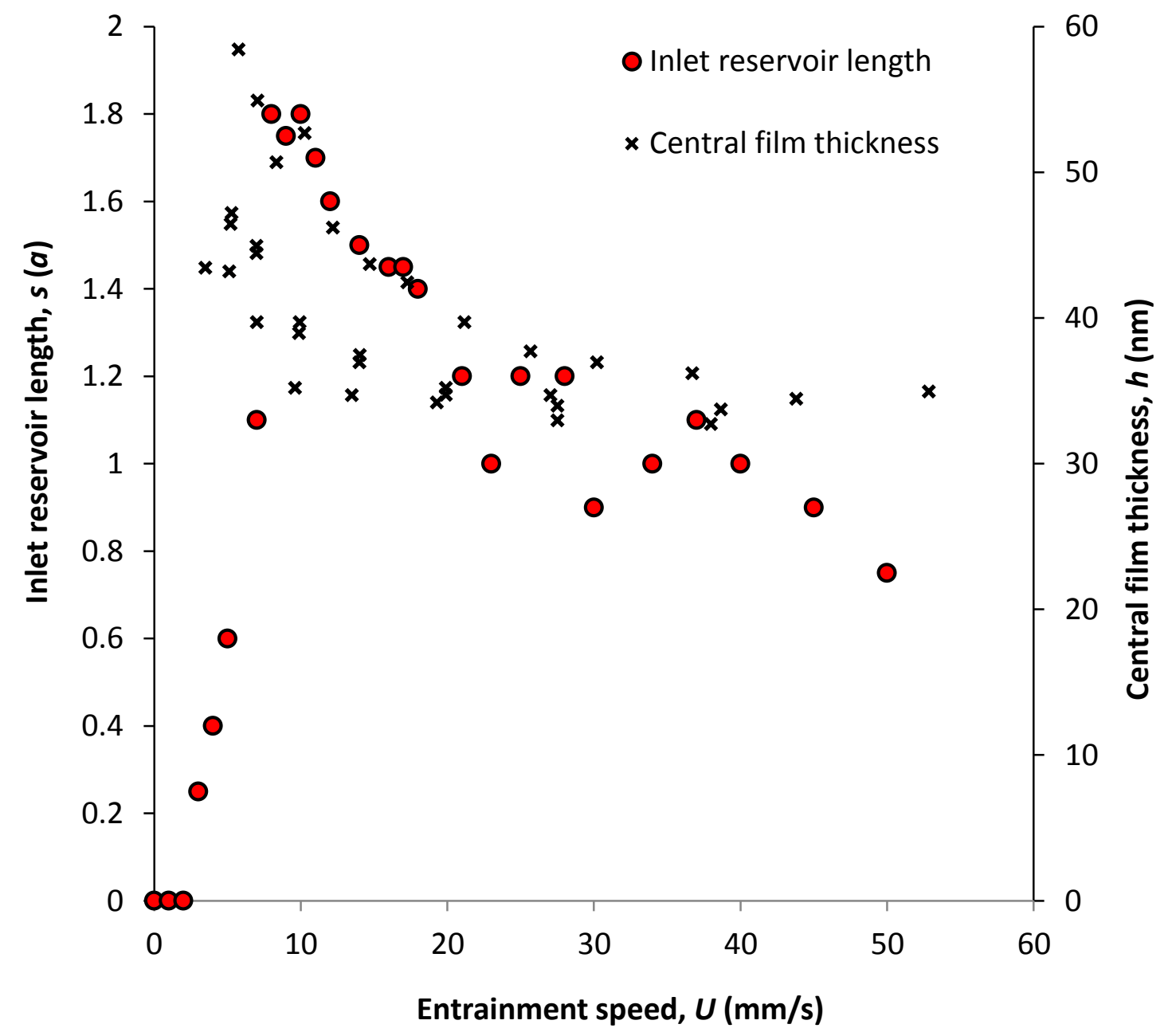

Figure 9. Measured film thickness and inlet reservoir (gel-phase) length plotted against entrainment speed. 


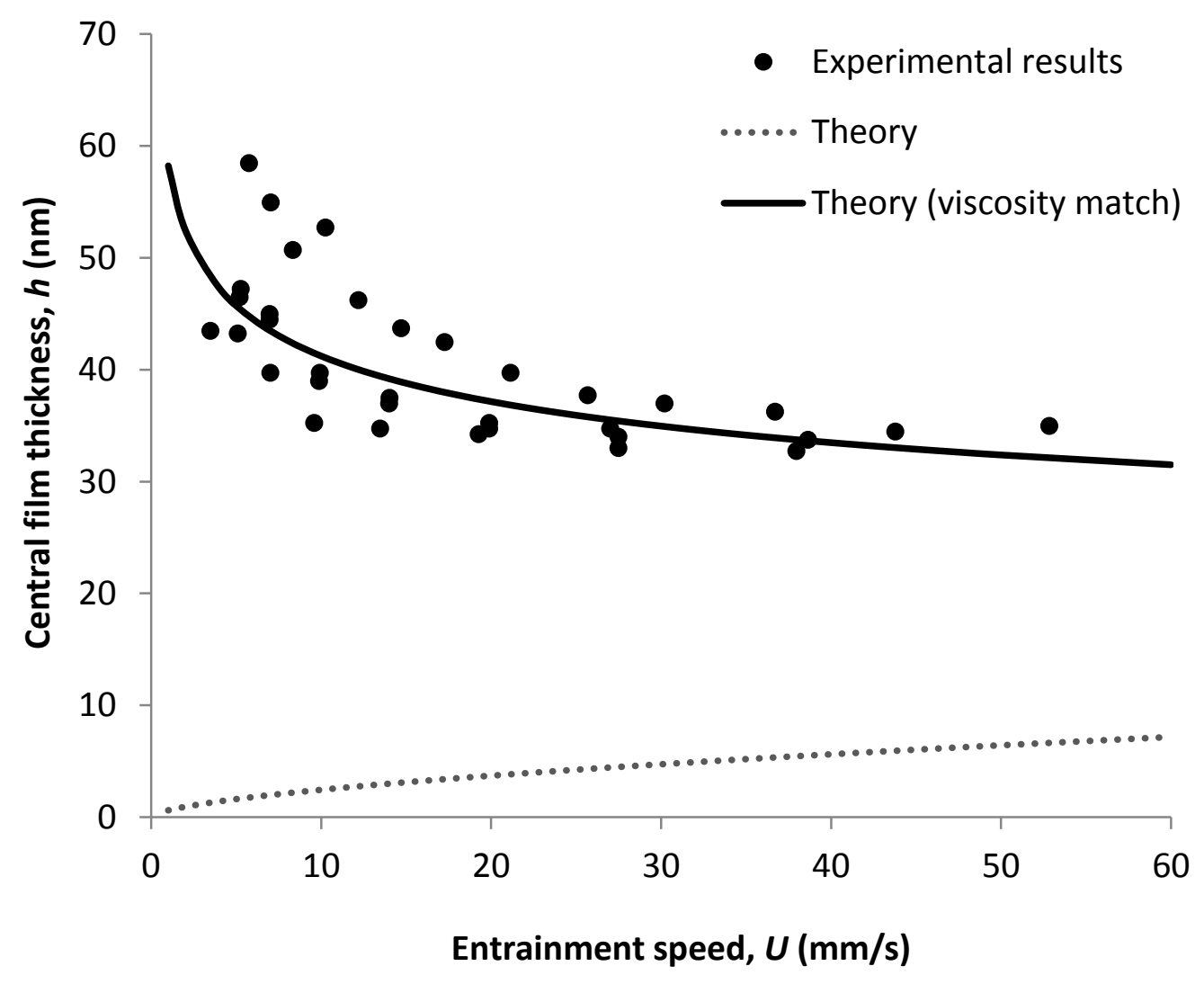

Figure 10. Comparison of measured and predicted film thickness (Newtonian and shearthinning models) plotted against entrainment speed.

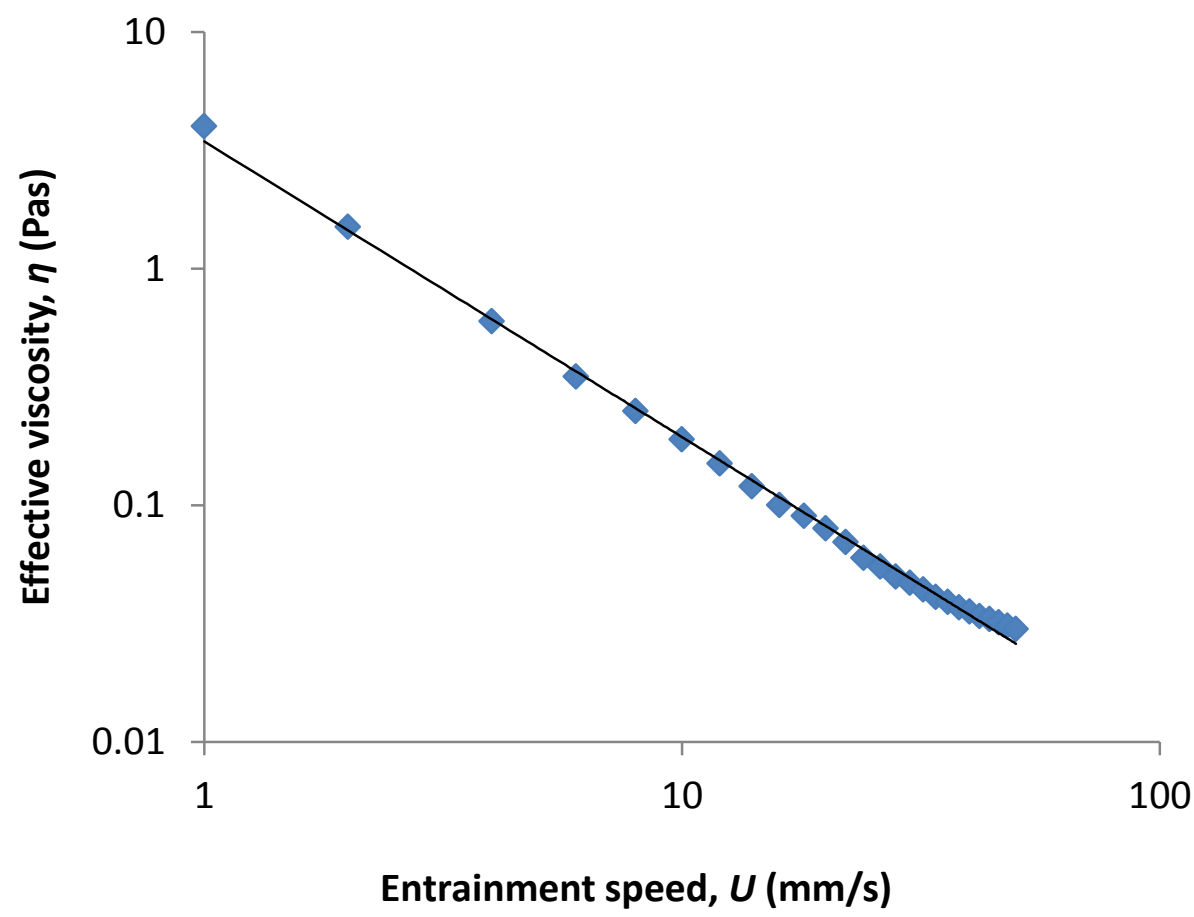

Figure 11. Effective viscosity (estimated from film thickness results) plotted against entrainment speed 